\title{
Is determination of transition zone volume by transrectal ultrasound in patients with clinically benign prostatic enlargement sufficiently reliable in the clinical setting?
}

\author{
Tomasz Szopinski ${ }^{1}$, Tomasz Golabek ${ }^{1}$, Andrzej Borówka ${ }^{2}$, Piotr Chłosta ${ }^{1}$ \\ ${ }^{1}$ Department of Urology, Jagiellonian University Medical College, Krakow, Poland \\ ${ }^{2} 1^{\text {st }}$ Department of Urology of the Postgraduate Medical Education Centre, European Health Centre, Otwock, Poland
}

Videosurgery Miniinv 2014; 9 (3): 398-403

DOI: $10.5114 /$ wiitm.2014.43128

\begin{abstract}
Introduction: Controversies exist regarding the accuracy of transrectal ultrasound (TRUS) determination of transition zone volume (TZV) when compared with enucleated adenoma weight.

Aim: To determine the accuracy and reliability of measurements of the TZV by TRUS, by comparing preoperative radiological findings with the enucleated prostate adenoma volume, measured by fluid displacement volumetry (FDV), after retropubic prostatectomy performed by the Millin method, and, moreover, to evaluate changes in the surgical capsule size in the intermediate postoperative period.

Material and methods: We measured TZV preoperatively using TRUS and postoperatively with FDV in 112 patients who underwent retroperitoneal prostatectomy for benign prostatic hyperplasia (BPH).

Results: The TRUS volume correlated well with specimen volumes $(r=0.945, p<0.0001)$. The median (quartile 1 , quartile 3) absolute error was $7.35 \mathrm{ml}(4.15 \mathrm{ml}, 9.28 \mathrm{ml})$ and the median percent error was $9.12 \%$ (4.75\%, 14.98\%). Percent error, but not absolute error, was significantly related to TRUS TZV ( $p<0.001$ and 0.217 , respectively). Adenomas $>80 \mathrm{cc}$ were associated with lower percent error. The median volume of the residual prostate tissue measured 3.5 years after prostatectomy was $92.65 \mathrm{cc}(65.75 \mathrm{cc}, 109.58 \mathrm{cc})$, whereas the median volume of the surgical capsule, depending on the equation used for its calculation, was $24.80 \mathrm{cc}(16.25 \mathrm{cc}, 37.37 \mathrm{cc})$ and $31.43 \mathrm{cc}(23.14 \mathrm{cc}, 43.32 \mathrm{cc})$. Conclusions: The TRUS TZV correlated well with values determined by FDV. It can be reliably used in clinical management of $B P H$.
\end{abstract}

Key words: simple prostatectomy, transition zone volume, transrectal ultrasound, benign prostatic enlargement.

\section{Introduction}

Although the transition zone (TZ) of the prostate accounts for less than $5 \%$ to $10 \%$ of the prostate gland volume, its significance for both benign and malignant pathologies cannot be underestimated [1, 2]. It is the exclusive site of origin of benign prostatic hyperplasia with a minor contribution from the periurethral glands $[1,3]$. The transition zone also has clinical relevance in patients with prostate cancer and its evaluation may be useful for diagnosis, staging, and treatment planning $[4,5]$.

Moreover, the measurements of the transition zone volume (TZV), which corresponds to the volume of the adenoma, often determine the modality of treatment for benign prostatic hyperplasia (BPH), including minimally invasive techniques and surgical approach [6]. 
The entire prostate and TZ volumes are most commonly estimated with transrectal ultrasound (TRUS). However, TZV measurement is operator dependent [7, 8]. Moreover, controversies exist regarding the accuracy of TRUS determination of TZV when compared with enucleated adenoma weight. While some authors have reported significant discrepancies $[9,10]$ others have found no differences $[11$, 12]. However, as all studies included a limited number of patients, some analysed specimens were only obtained following transurethral resection of the prostate or after suprapubic prostatectomy, or TZV determined by TRUS was compared with measured weight, the conclusions reached could have been adversely affected.

\section{Aim}

Therefore, the aim of this study was to determine the accuracy and reliability of measurements of TZV by transrectal ultrasound, by comparing preoperative radiological findings with the enucleated prostate adenoma volume, measured by fluid displacement volumetry, after retropubic prostatectomy performed by the Millin method. Moreover, we evaluated the residual prostate tissue by TRUS 3.5 years after the procedure to assess changes in its volume in the intermediate postoperative period.

\section{Material and methods}

The study was approved by the local research ethics committee, and all the procedures were carried out in accordance with the Helsinki Declaration of 1975, as revised in 1983. One hundred and twelve consecutive patients, who underwent retropubic prostatectomy (Millin operation) for histologically confirmed BPH, were included. All patients underwent TRUS before surgery, performed using a model 3535 Brüel and Kjaer (Denmark) system with a $7 \mathrm{MHz}$ multiplanar transrectal transducer. The prostate was scanned in the transverse and sagittal planes with the subjects in the left lateral decubitus position. Preoperative and postoperative prostate volumes were determined using the formula for a prolate ellipsoid $(\pi / 6 \times$ width $\times$ length $\times$ height) [13]. The width was the longest section on the transverse scan, length was the greatest anteroposterior distance on a sagittal scan, and height was the longest cephalic to caudal dimension in the sagittal plane.
The transverse, anteroposterior, and longitudinal dimensions of the transition zone were measured in the same planes in which total prostate dimensions were determined. The width of the TZ was measured starting from the inner part of the capsule, the length from the inner part of the capsule to the clear limit of the TZ at the verumontanum, and height from the bladder neck to the clear inferior limit. The volume of the TZ was estimated using the prolate ellipsoid formula.

The volume of the enucleated prostate adenoma was assessed immediately after the surgery using fluid displacement volumetry [14]. For this, the prostate adenoma specimen was placed in a transparent container filled with normal saline solution and the initial and final volumes were measured. The prostate volume was equal to the difference between final and initial volumes.

The volume of residual prostate tissue immediately after surgery, i.e. the surgical capsule, was calculated using two equations: i) by subtracting TZ volume from preoperative prostate volume measured by TRUS and ii) by subtracting prostate adenoma volume measured by fluid displacement volumetry from preoperative prostate volume determined by transrectal ultrasound.

To gauge the accuracy of TRUS volume, for estimating specimen volume determined by fluid displacement volumetry we calculated the absolute error using the formula: TRUS TZ volume (TZV) - adenoma volume measured by fluid displacement volumetry (AV). Similarly, the percent error in measurement was calculated using the following formula: $[\mathrm{TZV}-\mathrm{AV}] / \mathrm{AV} \times 100 \%$.

\section{Statistical analysis}

Since data in the studied group were not distributed in a Gaussian manner, the difference in median prostate adenoma volume and median TZV was evaluated by the Mann-Whitney $U$ test. A $p$ value of $<0.05$ was considered statistically significant. The Statistical Package for the Social Sciences (SPSS) was used for all statistical analyses.

Spearman's correlation coefficient and linear regression analysis were applied to determine the correlation of preoperative and postoperative prostate gland with TZV measured by the immersion method. The degree of association of the enucleated adenoma volume, measured by the immersion method with estimated transrectal ultrasound vol- 
ume of the transition zone, was also calculated by Spearman's correlation coefficient. The dependence of prostate adenoma volume, evaluated by the immersion method, on TZV was evaluated by linear regression analysis. Spearman's correlation coefficient and simple linear regression were used to determine the correlation of residual prostate tissue calculated immediately postoperatively with resid-

Table I. Patient demographics, TRUS volume, specimen volume, absolute and percent errors

\begin{tabular}{|lcc|}
\hline Variable & $N$ & Value \\
\hline Age, mean \pm SD & 112 & $70.58 \pm 5.93$ \\
\hline Median TPV (Q1, Q3) & 112 & $114.34(96.38,130.13)$ \\
\hline Median TZV (Q1, Q3) & 112 & $81.65(70.53,99.90)$ \\
\hline Median AV (Q1, Q3) & 112 & $80.00(61.00,93.00)$ \\
\hline Median TPV3 (Q1, Q3) & 112 & $92.65(65.75,109.58)$ \\
\hline$p$ Value TZV vs. AV & 112 & $<0.001$ \\
\hline Median TZV-AV (Q1, Q3) & 112 & $7.35(4.15,9.28)$ \\
\hline Median TPV-AV (Q1, Q3) & 112 & $31.43(23.14,43.32)$ \\
\hline Median TPV-TZV (Q1, Q3) & 112 & $24.80(16.25,37.37)$ \\
\hline TZV/AVx100\% & 112 & $91.65(86.98,95.47)$ \\
\hline TPV/AVx100\% & 112 & $70.05(59.73,78.70)$ \\
\hline TPV3/AVx100\% & 112 & $85.73(70.48,106.41)$ \\
\hline $\begin{array}{l}\text { Absolute error } \\
\text { (entire group) }\end{array}$ & 112 & $7.35(4.15,9.28)$ \\
\hline $\begin{array}{l}\text { Percent error } \\
\text { (entire group) }\end{array}$ & 112 & $9.12(4.75,14.98)$ \\
\hline Absolute error (TZ $\leq 80 c c)$ & 48 & $8.10(5.30,9.78)$ \\
\hline Percent error (TZ $\leq 80 c c)$ & 48 & $13.68(8.27,16.48)$ \\
\hline $\begin{array}{l}\text { Absolute error } \\
\text { (TZ > 80cc) }\end{array}$ & 64 & $6.55(2.93,9.00)$ \\
\hline Percent error (TZ > 80cc) & 64 & $6.69(3.07,9.68)$ \\
\hline $\begin{array}{l}p \text { Value for absolute } \\
\text { error } \leq 80 \text { cc vs. } \leq 80 c c\end{array}$ & 112 & 0.217 \\
\hline $\begin{array}{l}p \text { Value for percent } \\
\text { error } \leq 80 \text { cc vs. } \leq 80 c c\end{array}$ & 112 & $<0.001$ \\
\hline
\end{tabular}

Mean age is expressed in years; SD - standard deviation, SD is expressed in years, TPV - total prostate volume estimated preoperatively by TRUS, TZV - transition zone volume estimated preoperatively by TRUS, AV - adenoma volume determined by fluid displacement volumetry, TPV 3 - residual prostate tissue volume estimated by TRUS 3.5 years after retropubic prostatec tomy; TZV/AVX100\% - the median percent of TZV estimated preoperatively by TRUS that corresponds to adenoma volume determined by fluid displacement volumetry; TPV/AV $100 \%$ - the median percent of TPV estimated preoperatively by TRUS that corresponds to adenoma volume determined by fluid displacement volumetry; TPV $3 / A V \times 100 \%$ - the median percent of TPV 3 estimated by TRUS 3.5 years after retropubic prostatectomy that corresponds to adenoma volume determined by fluid displacement volumetry, vs. - versus, $n$-number of patients ual prostate measured 3.5 years following surgery. The difference in median prostate adenoma volume and median TZV was evaluated by the Mann-Whitney $U$ test.

\section{Results}

The median values of volumes determined in 112 patients are presented in Table I. The median (low quartile, high quartile) TZV measured by TRUS was $81.65 \mathrm{cc}(70.53 \mathrm{cc}, 99.90 \mathrm{cc})$, whereas the corresponding volume of the enucleated adenoma was $80.00 \mathrm{ml}(61.00 \mathrm{ml}, 93.00 \mathrm{ml})$. The difference between the estimate with TRUS and the surgical specimen was statistically significant $(p<0.001)$.

The median volume of the entire prostate determined by TRUS preoperatively was $114.34 \mathrm{cc}$ ( $96.38 \mathrm{cc}$, $130.13 \mathrm{cc}$ ). The median volumes of the surgical capsule calculated by subtraction of TZV from preoperative prostate volume measured by TRUS, and also by subtracting prostate adenoma volume, measured by fluid displacement volumetry, from preoperative prostate volume determined by transrectal ultrasound were 24.80 cc (16.25 cc, 37.37 cc) and 31.43 cc (23.14 cc, 43.32 cc), respectively.

The correlation between the TZV estimated by TRUS and by fluid displacement volumetry ( $r=$ $0.945, p<0.0001$, Figure 1) was statistically significant. The correlations between the adenoma volume measured by fluid displacement volumetry and the preoperative prostate volume estimated by TRUS $(r=0.633, p<0.0001$, Figure 2$)$, and between the

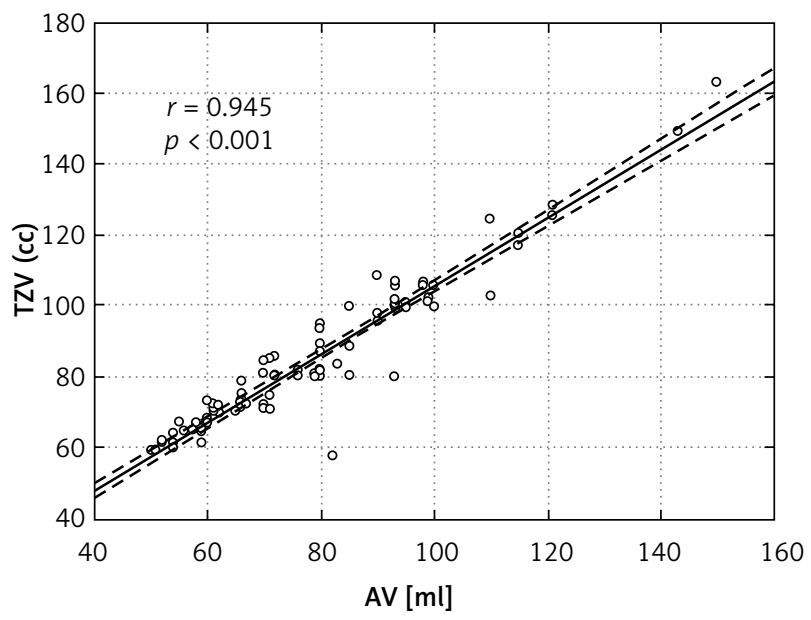

Figure 1. Scatterplot and line of best fit for TRUS TZV as function of specimen volume (AV) estimated by fluid displacement volumetry 


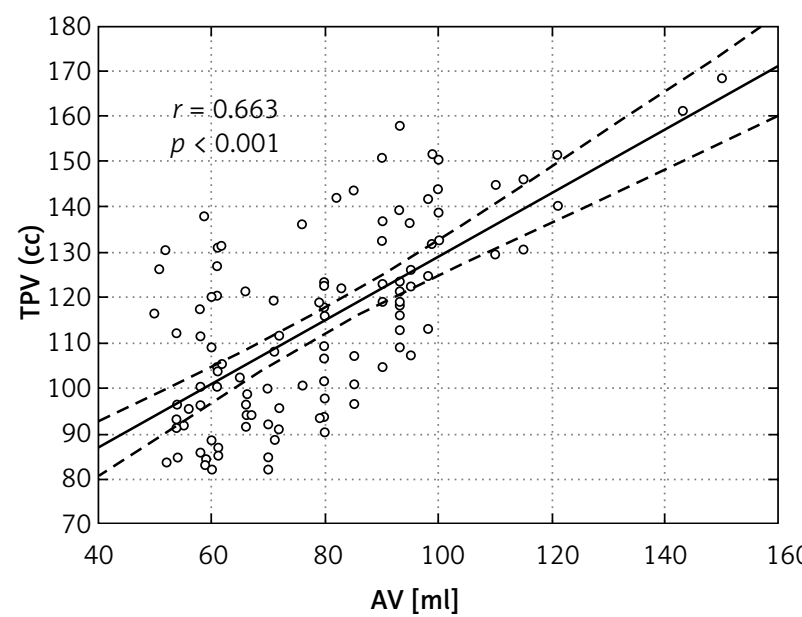

Figure 2. Scatterplot and line of best fit for TRUS total prostate volume (TPV) as function of specimen volume (AV) estimated by fluid displacement volumetry

adenoma volume measured by fluid displacement volumetry and residual prostate tissue determined by TRUS on average 3.5 years (42 months) $( \pm \mathrm{SD}=$ 6.51 months) after surgery $(r=0.407, p<0.0001$, Figure 3), were both statistically significant. No correlation was found between the surgical capsule calculated immediately after surgery and estimated by TRUS 3.5 years after retropubic prostatectomy.

Overall, median absolute error was $7.35 \mathrm{ml}$ $(4.15 \mathrm{ml}, 9.28 \mathrm{ml})$, and median percent error was $9.12 \%$ (4.75\%, 14.98\%). Percent error was significantly associated with TRUS TZV with higher TRUS volumes associated with lower percent error $(p<0.001)$. The greatest percent error was seen in patients with TRUS TZ volume of 80 cc or less (Figure 4).

\section{Discussion}

Accurate measurement of the prostate gland size is important in clinical settings as it determines BPH management and prostate cancer risk classification. The volume of the TZ of the prostate influences the choice of surgical technique in patients with symptomatic benign prostatic hyperplasia [6]. The TRUS has been the most commonly used method for TZV estimation. Although it remains a gold standard in determining prostate size in patients with benign disease, it is not, however, without inaccuracies $[7,8]$. The accuracy of prostate TZV estimations in patients with $\mathrm{BPH}$ has been a subject of several studies, but these have involved either small co-

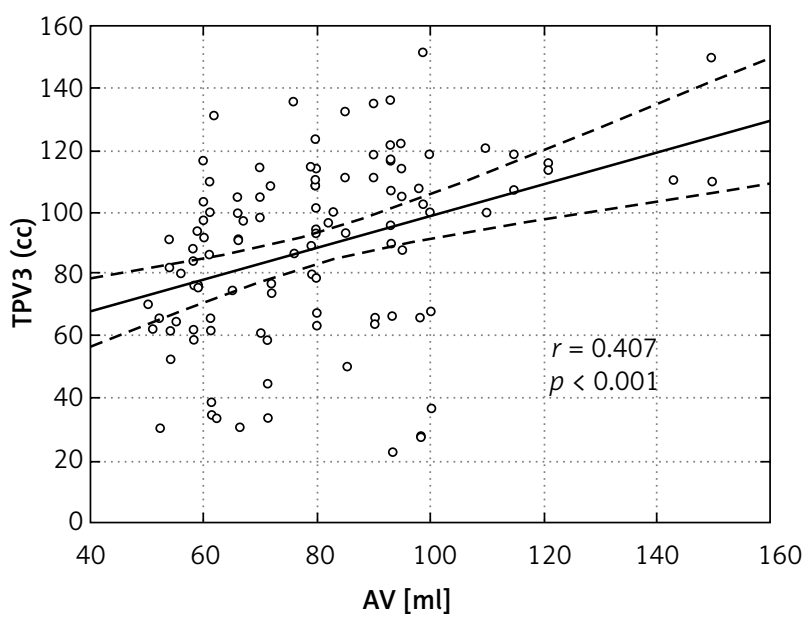

Figure 3. Scatterplot and line of best fit for residual tissue volume estimated by TRUS 3.5 years following surgery (TPV3) as function of specimen volume (AV) estimated by fluid displacement volumetry

horts, limiting their analyses to specimens obtained following transurethral resection of the prostate or after suprapubic prostatectomy, or compared TZV determined by TRUS with measured weight $[9,11$, 12]. Whereas prostate weight and volume are theoretically interchangeable since the specific gravity of prostate tissue is 1.05 , only the former is a true measurement and the latter is mathematically calculated [15]. Hence, there is a potential risk that conclusions drawn based on the above association

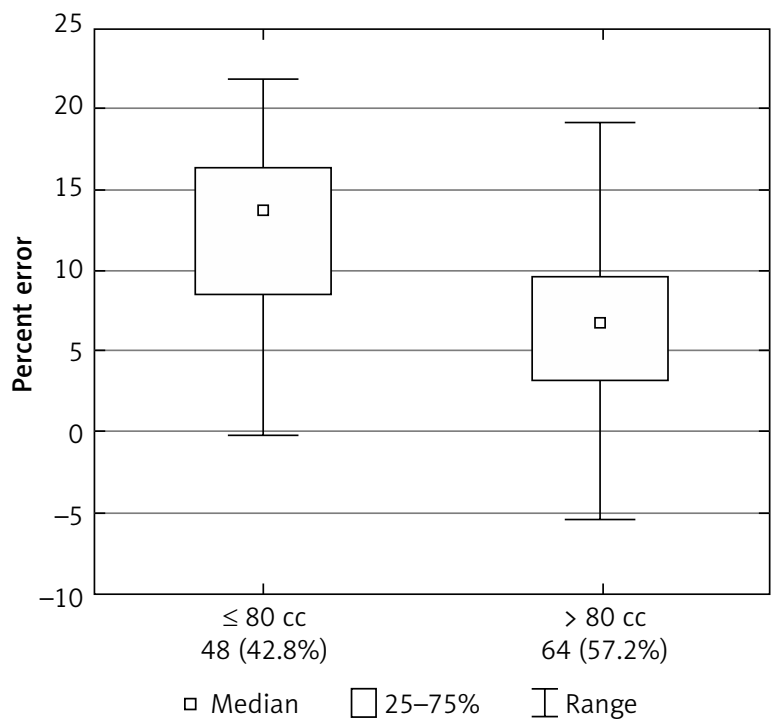

Figure 4. Box plot of percent error shows number and percent of patients per category for TRUS TZV of $\leq 80 \mathrm{cc}$ and $>80 \mathrm{cc}$ 
would be invalid. In the present study we sought to determine the general comparability of estimated volumes by TRUS when compared with volumes determined by fluid displacement volumetry.

In our cohort of 112 men who underwent retropubic prostatectomy for symptomatic $\mathrm{BPH}$, we found that TRUS TZV estimation correlated well with volume of adenoma specimen determined by fluid displacement volumetry $(r=0.945, p<0.0001)$.

Consistent with other reports, we found that TRUS volume accuracy (expressed in this study as percent error) depends on TRUS volume [16-18]. In the present study, men with smaller adenoma volume $(<80 \mathrm{cc})$ had the greatest percent error.

In a study by Matthews et al., TRUS volume overestimation was noted in glands smaller than $30 \mathrm{cc}$ and underestimation in larger than 50 cc [19]. Terris and Stamey noted that it may be more accurate to apply a prolate spheroid formula, for smaller glands (less than $80 \mathrm{~g}$ ), and use other equation for larger prostates [16].

In our study median TRUS TZV was $81.65 \mathrm{cC}$ (70.53 cc, $99.90 \mathrm{cc}$ ) and median retropubic prostatectomy specimen volume was $80.00 \mathrm{ml}(61.00 \mathrm{ml}$, $93.00 \mathrm{ml})$, giving a correction factor of (80.00/81.65) $=0.97$. In terms of clinical accuracy, the fact that the correction factor was not much different than 1.0 implies that TRUS volume can be used clinically without correction.

In addition, although percent error depended on TRUS volume, the absolute error was not affected by prostate size $(8.10 \mathrm{ml}$ and $6.55 \mathrm{ml}$ in $\leq 80 \mathrm{cc}$ and $>80 \mathrm{cc}$, respectively, $p=0.217)$. Therefore, TRUS can be used to estimate TZV regardless of its size.

In this study we also estimated volume of the residual tissue following retropubic prostatectomy (i.e. surgical capsule) and correlated it to the TRUS measurement performed 3.5 years after surgery. Median volume of the surgical capsule calculated by subtracting TZV from preoperative prostate volume both measured by TRUS, and by subtracting prostate adenoma volume measured by fluid displacement volumetry from preoperative prostate volume determined by TRUS, was 24.80 cc (16.25 cc, $37.37 \mathrm{cc})$ and $31.43 \mathrm{cc}(23.14 \mathrm{cc}, 43.32 \mathrm{cc})$, respectively. The difference between those two estimations was statistically significant $(p<0.001)$. Interestingly, median volume of the residual prostate tissue measured by TRUS 3.5 years after prostatectomy was $92.65 \mathrm{cc}$ (65.75 cc, 109.58 cc). Hence, it was approximately
3 times larger than the calculated volume of tissue at the time of surgery, irrespective of whichever equation we used. However, there are two noteworthy limitations of this study that could affect estimation of volume of the residual prostate tissue in the intermediate follow-up period. These include the exclusive use of calculation-based determination of volume of the surgical capsule without TRUS measurements in the early period after surgery which would be long enough to allow for oedema to subside but not allow for bio-mechanical processes to develop and affect measurements, also not taking into account the volume of the resected bed while estimating the size of the residual tissue in the intermediate follow-up period.

However, we believe that such a significant increase in median volume of the residual tissue observed 3.5 years after prostatectomy as compared to the preoperational values cannot simply be explained by inaccuracy in the method we used to estimate volume of the surgical capsule prior to surgery. Moreover, since the Millin retropubic prostatectomy was performed in all cases, a complete enucleation of the adenoma is more than expected. Also, significant neohyperplasia of the residual tissue, that could result in a marked increase of its volume, seems to be unlikely, as the enucleation was complete and the follow-up period was not long [18]. Therefore, occurrence of bio-mechanical processes in the residual tissue need to be considered. These may include relaxation of collagen fibres which are an important component of the prostatic peripheral zone tissue [20].

\section{Conclusions}

The TRUS volume correlated well with values determined by fluid displacement volumetry. The percent error in TRUS volume estimation depends on the measured volume with the highest accuracy in men with TZV > 80 cc. However, absolute error was not affected by volume. Therefore, TRUS estimation of TZV is reliable and can be used clinically without correction. Further research should look into processes occurring within residual prostate tissue and their significance for clinical practice.

\section{References}

1. McNeal JE. Normal histology of the prostate. Am J Surg Pathol 1988; 12: 619-33. 
2. Chłosta PL, Drewa T, Jaskulski J, et al. Bladder neck preservation during classic laparoscopic radical prostatectomy - point of technique and preliminary results. Videosurgery Miniinv 2012; 7: 89-95.

3. McNeal JE. Normal anatomy of the prostate and changes in benign prostatic hypertrophy and carcinoma. Semin Ultrasound CT MR 1988; 9: 329-34.

4. Myschetzky PS, Suburu RE, Kelly BS Jr, et al. Determination of prostate gland volume by transrectal ultrasound: correlation with radical prostatectomy specimens. Scand J Urol Nephrol Suppl 1991; 137: 107-11.

5. Zlotta AR, Djavan B, Marberger M, Schulman CC. Prostate specific antigen density of the transition zone: a new effective parameter for prostate cancer prediction. J Urol 1997; 157: 1315-21.

6. Oelke M, Bachmann A, Descazeaud A, et al.; members of the European Association of Urology (EAU) Guidelines Office. Guidelines on management of male lower urinary tract symptoms (LUTS), incl. benign prostatic obstruction (BPO). In: EAU Guidelines, edition presented at the 26th EAU Annual Congress, Vienna 2011.

7. Bazinet M, Karakiewicz PI, Aprikian AG, et al. Reassessment of nonplanimetric transrectal ultrasound prostate volume estimates. Urology 1996; 47: 857-62.

8. Tong S, Cardinal HN, McLoughlin RF, et al. Intra- and inter-observer variability and reliability of prostate volume measurement via two-dimensional and three-dimensional ultrasound imaging. Ultrasound Med Biol 1998; 24: 673-81.

9. Baltaci S, Yagci C, Aksoy $\mathrm{H}$, et al. Determination of transition zone volume by transrectal ultrasound in patients with clinically benign prostatic hyperplasia: agreement with enucleated prostate adenoma weight. J Urol 2000; 164: 72-5.

10. Cabello Benavente R, Jara Rascón J, Monzó J, et al. Volume determinations of the whole prostate and of the adenoma by transrectal ultrasound: correlation with surgical specimen. Actas Urol Esp 2006; 30: 175-80.

11. Zlotta AR, Djavan B, Damoun M, et al. The importance of meas uring the prostatic transition zone: an anatomical and radiological study. BJU Int 1999; 84: 661-6.

12. Milonas D, Matjosaitis A, Jievaltas M. Transition zone volume measurement - is it useful before surgery for benign prostatic hyperplasia? Medicina (Kaunas) 2007; 43: 792-7.

13. Aarnink RG, de la Rosette JJ, Debruyne FM, Wijkstra H. Formula-derived prostate volume determination. Eur Urol 1996; 29: 399-402.

14. Aarnik R, Wijkstraa H. Volume measurement. In: The measurement, instrumentation, and sensors: handbook. Webster JG (ed.) , CRC Press LLC and Springer-Verlag GmbH \& Co. KG 1999; 13.1-13-6.

15. Watanabe $H$, Igari D, Tanahashi Y, et al. Measurements of size and weight of prostate by means of transrectal ultrasonotomography. Tohoku J Exp Med 1974; 114: 277-85.

16. Terris MK, Stamey TA. Determination of prostate volume by transrectal ultrasound. J Urol 1991; 145: 984-7.

17. Sajadi KP, Terris MK, Hamilton RJ, et al. Body mass index, prostate weight and transrectal ultrasound prostate volume accuracy. J Urol 2007; 178: 990-5.
18. Ballesteros Sampol JJ, Royo Lázaro J, Lloreta Trull J. Histomorphometric findings in the residual prostate and prostatic hollow after retropubic surgery for BPH: (I) baseline data obtained from the last 70 consecutive cases. Arch Esp Urol 2007; 60: 69-76.

19. Matthews GJ, Motta J, Fracehia JA. The accuracy of transrectal ultrasound prostate volume estimation: clinical correlations. J Clin Ultrasound 1996; 24: 501-5.

20. Zhang Y, Nojima S, Nakayama H, et al. Characteristics of normal stromal components and their correlation with cancer occurrence in human prostate. Oncol Rep 2003; 10: 207-11.

Received: 27.11.2013, accepted: 26.01.2014 